Boise State University

ScholarWorks

$5-20-2020$

\title{
Poly(carboxypyrrole)s That Depolymerize from Head to Tail in the Solid State in Response to Specific Applied Signals
}

Hyungwoo Kim

Chonnam National University

Adam D. Brooks

Boise State University

Anthony D. DiLauro

Boise State University

Scott T. Phillips

Boise State University

This is an open access article published under an ACS AuthorChoice License, which permits copying and redistribution of the article or any adaptations for non-commercial purposes. This document was originally published in Journal of the American Chemical Society by the American Chemical Society. Copyright restrictions may apply. doi: 10.1021/jacs.0c02774 


\title{
Poly(carboxypyrrole)s That Depolymerize from Head to Tail in the Solid State in Response to Specific Applied Signals
}

\author{
Hyungwoo Kim,* Adam D. Brooks, Anthony M. DiLauro, and Scott T. Phillips*
}

Cite This: J. Am. Chem. Soc. 2020, 142, 9447-9452

Read Online

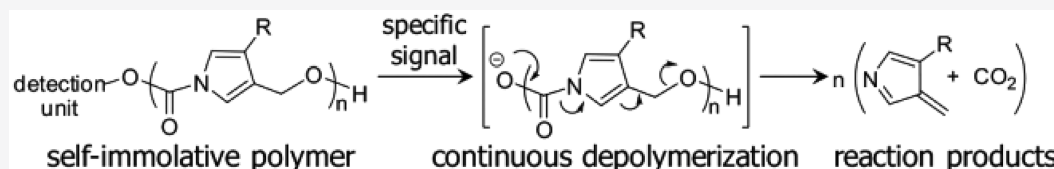

ABSTRACT: This Article describes the design, synthesis, and analysis of a new class of polymer that is capable of depolymerizing continuously, completely, and cleanly from head to tail when a detection unit on the head of the polymer is exposed to a specific applied signal. The backbone of this polymer consists of 1,3-disubstituted pyrroles and carboxy linkages similar to polyurethanes. Diverse side chains or reactive end-groups can be introduced readily, which provides modular design of polymer structure. The designed depolymerization mechanism proceeds through spontaneous release of carbon dioxide and azafulvene in response to a single triggering reaction with the detection unit. These poly(carboxypyrrole)s depolymerize readily in nonpolar environments, and even in the bulk as solid-state plastics.

\section{INTRODUCTION}

Self-immolative, unzipping, and $\mathrm{CD}_{\mathrm{r}}$ (continuous depolymerization in response to a reaction-based detection event) all are terms given to a small class of stimuli-responsive polymers that have the unique ability to depolymerize continuously and completely from end-to-end when exposed to one equivalent of a specific applied stimulus. These polymers typically contain a detection unit at their head, where the detection unit stabilizes the polymer backbone and prevents spontaneous depolymerization. ${ }^{1}$ Once this detection unit reacts with a specific applied signal, it is cleaved from the end of the polymer, which triggers the polymer to depolymerize completely to either monomers or predetermined small molecules. The detection unit can be changed easily to alter the signal to which the polymer responds, which enables mixing and matching of detection units and applied signals to provide selective depolymerization to a desired signal under mild conditions. ${ }^{2}$

Such triggered depolymerization offers the potential for causing rapid changes in materials because only a single reaction is necessary to induce a polymer to depolymerize. Likewise, self-immolative $\mathrm{CD}_{\mathrm{r}}$ polymers enable highly sensitive stimuli-responsive materials, because only a single equivalent of the applied signal is required to induce large-scale changes in the physical properties of materials. ${ }^{3}$ Similarly, a burst of depolymerization products that are induced by a single triggering event leads to chemical amplification of defined products.

Examples of such polymers include poly(benzyl carbamate)$\mathrm{s}^{5}$ poly(quinone methide)s, ${ }^{6}$ poly(phthalaldehyde)s, ${ }^{7}$ polyglyoxylates, ${ }^{8}$ and closely related polyglyoxamides, ${ }^{9}$ as well as polycarbamates and polythiocarbonates that depolymerize via intramolecular cyclization reactions. ${ }^{10}$ There are also similar classes of polymers that depolymerize continuously when the backbone of the polymer is fragmented, either by photolysis, specific chemical signals, or mechanical force, or by incorporation of a triggered catalyst, such as a photoacid. ${ }^{11}$

Self-immolative $\mathrm{CD}_{\mathrm{r}}$ polymers, in particular, offer a unique opportunity for designing new generations of soft materials that are capable of depolymerizing selectively and controllably, at room temperature, when they are no longer needed. Such materials could enable rapid, energy-efficient recycling of plastics and may provide a solution to eliminating the accumulation of plastic waste in the environment. ${ }^{12}$

To realize this promise, new classes of self-immolative $C_{r}$ polymers are needed that are capable of depolymerizing rapidly and completely in the solid state, when triggered as macroscopic materials. Poly(phthalaldehyde) and chlorinated poly(phthalaldehyde ${ }^{13}$ have this capability, but linear poly(phthalaldehyde)s are chemically unstable when stored, and phthalaldehyde-based monomers are known to be toxic. Select derivatives of poly(quinone methide)s depolymerize as macroscopic plastics when triggered, albeit slowly at room temperature, which limits their potential. ${ }^{14}$

This lack of self-immolative $\mathrm{CD}_{\mathrm{r}}$ polymers that depolymerize in the solid state is a deficiency that we aim to correct with this

Received: March 10, 2020

Published: April 24, 2020 


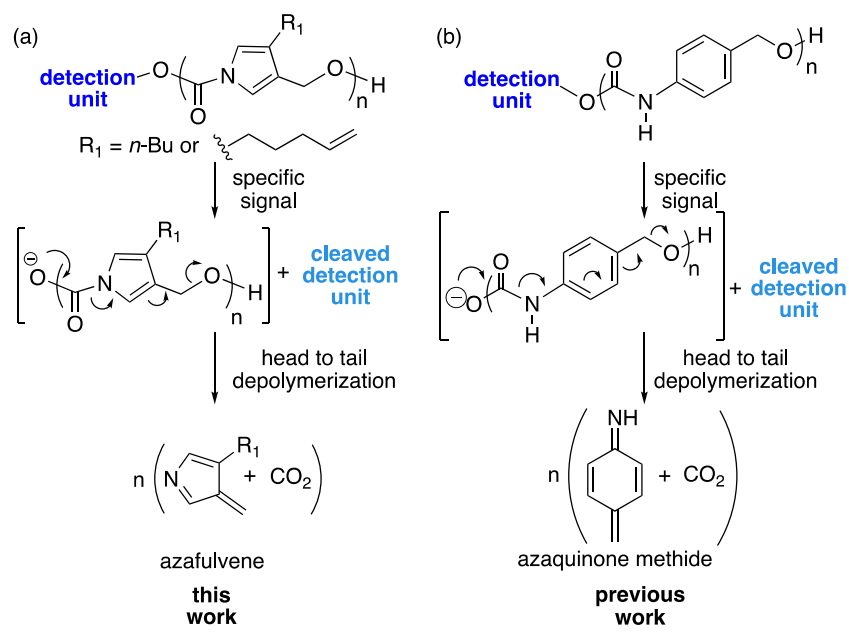

Figure 1. Chemical structure and proposed triggered depolymerization mechanism of (a) self-immolative poly(carboxypyrrole)s in comparison with (b) self-immolative poly(benzyl carbamate) s. $^{5}$

report. Herein, we describe the design and synthesis of a new class of self-immolative polymer (Figure 1a) that is capable of depolymerizing completely from head to tail in the solid state when triggered by specific applied signals that react with the detection units on the polymers. We further demonstrate that this new polymer class (i.e., poly(carboxypyrrole)) is able to function as a macroscopic plastic. Given its ability to depolymerize in the solid state, it offers a starting point for creating selectively depolymerizable coating materials, adhesives, elastomers, thermoplastics, thermosets, and foams. ${ }^{15}$

\section{RESULTS AND DISCUSSION}

The new self-immolative $\mathrm{CD}_{\mathrm{r}}$ polyurethane is depicted in Figure 1a. This polymer is designed to depolymerize through a mechanism distinct from its polymerization mechanism and thus releases carbon dioxide and azafulvene from each repeating unit as the polymer unzips. Formation of carbon dioxide prevents the reverse reaction, and azafulvene enables the polymer to convert entirely to a well-defined pyrrole derivative upon complete depolymerization.

We selected pyrrole as the core for each repeating unit because elimination of the next repeating unit, once triggered, should proceed through a mechanism that is similar to the proposed 1,6-azaquinone methide elimination mechanism for depolymerization of poly(benzyl carbamate)s (Figure $1 \mathrm{~b}) .^{5}$ Our early work with poly(benzyl carbamate)s demonstrated that the formation of azaquinone methide is exceedingly slow in all contexts except when the polymers are dissolved in highly polar solvents, such as DMSO or water. ${ }^{16}$ Using small molecule model systems, we learned that reducing the aromaticity of the central ring from benzene to naphthalene provided a $23 \%$ reduction in aromaticity relative to benzene, and a corresponding $12 \times$ increase in the rate of triggered release of the pendant benzylic group. Because pyrrole is predicted to be $40 \%$ less aromatic than benzene (Table S1), ${ }^{17}$ we reasoned that a pyrrole-based self-immolative polymer might enable rapid azaquinone methide-like depolymerization in nonpolar environments, and even in the solid state.

To test this hypothesis, we prepared functionalized derivatives of poly(carboxypyrrole)s using two orthogonal routes (Scheme 1). One route employed the van Leusen pyrrole synthesis, which uses $\beta$-substituted acrylic acids (e.g.,
Scheme 1. Two Synthetic Routes to Functionalized Monomers $^{a}$

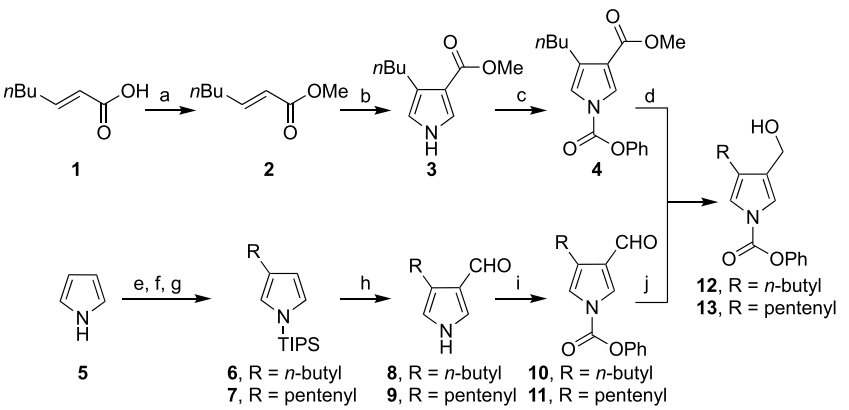

${ }^{a}$ Reagents and conditions: (a) MeI, $\mathrm{KHCO}_{3}, \mathrm{DMF}$ (97\%); (b) TosMIC, NaH, THF (85\%); (c) PhOCOCl, DIEA, DMAP, MeCN; (d) DIBAL, THF (43\% over two steps); (e) NaH, TIPSCl, THF (87\%); (f) NBS, THF; (g) $n$-BuLi, iodocompounds (1-iodobutane for 6, 5-iodo-1-pentene for 7), THF; (h) Vilsmeier reagent (8, 17\% over three steps; 9, 18\% over three steps); (i) PhOCOCl, DIEA, DMAP, $\mathrm{MeCN}$; (j) $\mathrm{NaBH}_{4}, \mathrm{AcOH}$, benzene $(\mathbf{1 0}, 81 \%$ over two steps; 11, $71 \%$ over two steps).

reagent 1 ) to introduce functionality on the 4-position of the pyrrole monomer. The second route incorporates functionality at the 4-position via an alkylation reaction (e.g., pyrrole 6) using a lithiated pyrrole. The choice of synthetic route depends on the compatibility of the desired functional group at the 4position with the reactions to be used for its introduction. If the desired functionality at the 4-position is compatible with both routes, then the van Leusen pyrrole synthesis is preferred over the ring substitution route because the former provides monomer in four steps (35\% overall yield for $n$-butyl derivative 12), while the latter requires six steps ( $11 \%$ overall yield for 12).

The pyrrole monomers are bifunctional, containing a phenyl carbamate on the $\mathrm{N}$-terminus and an alcohol nucleophile at the pyrrylic position. Polymerization of monomers at $60{ }^{\circ} \mathrm{C}$ using catalytic quantities of 1,8-diazabicycloundec-7-ene (DBU) provided access to polymer, which was directly end-capped by addition of an alcohol before the polymer was isolated (Scheme 2). These conditions were reasonably efficient ( $\sim 50-60 \%$ yields) and provided polymers that contained $>20$ repeating units. We noted that stoichiometric quantities of DBU (relative to the monomer) were ineffective at generating polymer (only small molecules remained), ${ }^{16}$ which is not surprising given the intricacies of organo-catalyzed polymerization reactions. ${ }^{18}$

The end-capping reaction places the detection unit on the head of the polymer. Examples of select detection units are indicated in Scheme 2 as polymers 14-17. Polymers 14 and 17 contain aryl boronate detection units that are designed to respond selectively to hydrogen peroxide. Polymer 15 contains an allyl carbonate detection unit for responding to palladium(0), and polymer $\mathbf{1 6}$ is end-capped with an isopropyl carbonate to serve as a control because it should not react with either hydrogen peroxide or palladium $(0)$.

Selective depolymerization of the poly(carboxypyrrole)s is achieved (up to $\sim 75 \%$ completion) by exposing a polymer containing an appropriate detection unit to its intended signal. For example, exposure of polymer 14 (aryl boronate detection unit) dissolved in THF to aqueous hydrogen peroxide at $23{ }^{\circ} \mathrm{C}$ resulted in complete depolymerization, as indicated by gel permeation chromatography (GPC) and ${ }^{1} \mathrm{H}$ NMR spectros- 


\section{Scheme 2. Polymerization of Monomers ${ }^{a}$}

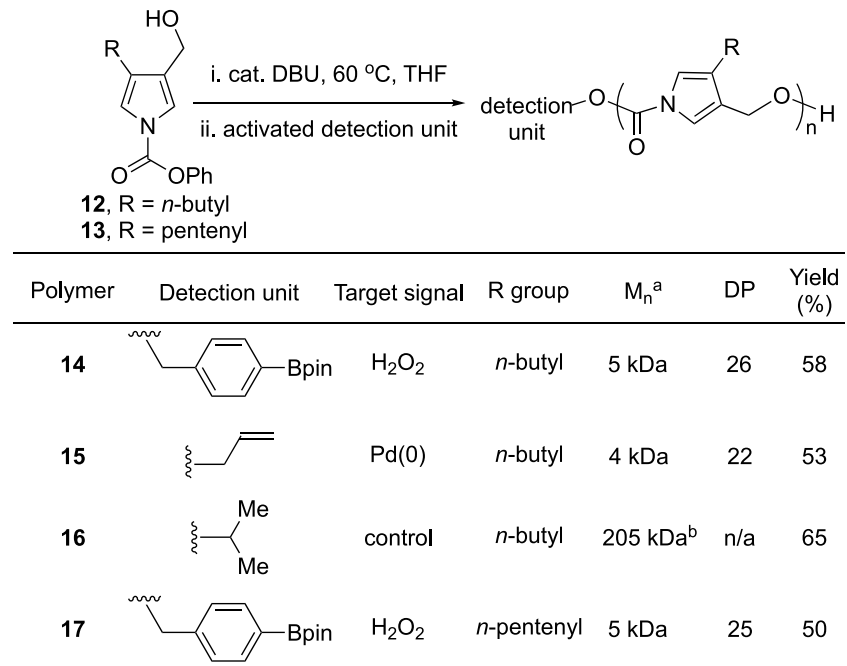

a (a) $M_{\mathrm{n}}$ and degree of polymerization (DP) values were estimated by end-group analysis using ${ }^{1} \mathrm{H}$ NMR data. Data obtained from gel permeation chromatography (GPC) equipped with a multiangle light scattering detector are available in the Supporting Information. We suspect that the short chain lengths of these oligomers may be manifesting in an overestimate of chain length by GPC. (b) The endgroup was not visible in the ${ }^{1} \mathrm{H}$ NMR spectrum for 16 , so the $M_{\mathrm{n}}$ value is reported from data obtained via the GPC/multiangle light scattering technique. We are not reporting a DP value for this oligomer because we could not perform end-group analysis like we did with the other example oligomers.

copy (Figure 2a). GPC allowed us to qualitatively track the disappearance of the polymer peak, which has a retention time of 16 min (inset in Figure 2a; Figure S2), and ${ }^{1} \mathrm{H}$ NMR analysis yielded quantitative measurements of depolymerization by providing the ratio between the methylene hydrogens on each repeating unit and the aldehyde hydrogen on $N, N$-dimethylformamide, which was used as an internal standard.

NMR analysis of $\mathbf{1 4}$ when treated with hydrogen peroxide showed not only the disappearance of the polymer methylene peak but also the disappearance of the pyrrole peaks $(\delta=7.01$ and $7.35 \mathrm{ppm}$ ) and concomitant formation of the corresponding small molecule peaks ( $\delta=6.62$ and $7.05 \mathrm{ppm}$ ) (Figure S1). The ${ }^{1} \mathrm{H}$ NMR data also revealed a 10 min induction period prior to measurable depolymerization (Figure 2a). We attribute this induction period either to a slow oxidative cleavage reaction between hydrogen peroxide and the aryl boronate or to quinone methide-mediated release of the head of the polymer. Once released, the polymer undergoes rapid head-to-tail depolymerization, which is complete within 30 min of exposure to hydrogen peroxide.

The desired depolymerization response is critical, but the absence of undesired, nonspecific degradation is equally important. When 14 is dissolved in THF and exposed to triethylamine for $7 \mathrm{~d}$ in the absence of hydrogen peroxide, no depolymerization or degradation is observed by ${ }^{1} \mathrm{H}$ NMR (Figure S3). Similarly, if control polymer 16 (containing an isopropyl carbonate end-cap) is exposed to hydrogen peroxide and triethylamine in THF, no depolymerization is detected, even after $24 \mathrm{~h}$ (GPC analysis, Figure $2 \mathrm{~b} ;{ }^{1} \mathrm{H}$ NMR analysis, Figure S11).
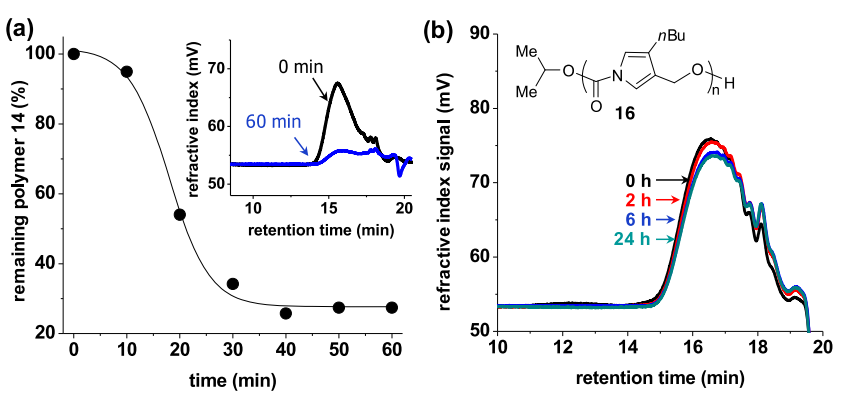

Figure 2. Selective triggered head-to-tail depolymerization of polymer 14 in THF- $d_{8}$ when exposed to aqueous hydrogen peroxide (10 equiv relative to detection unit) and triethylamine $(10 \text { equiv })^{19}$ at $23^{\circ} \mathrm{C}$. (a) The percentage of remaining polymer was calculated from integrated ${ }^{1} \mathrm{H}$ NMR data relative to an internal standard (DMF). The inset shows the results of GPC analysis of $\mathbf{1 4}$ exposed to hydrogen peroxide under identical conditions. (b) GPC spectra of control polymer 16 exposed to hydrogen peroxide and triethylamine for $24 \mathrm{~h}$.

Altering the detection unit changes the signal to which the polymer responds, as designed. For example, exposure of polymer 15 (allyl carbonate detection unit) dissolved in $\mathrm{CDCl}_{3}$ to 2 equiv of $\mathrm{Pd}\left(\mathrm{PPh}_{3}\right)_{4}$ causes complete depolymerization of the polymer in approximately $5 \mathrm{~min}$, as measured both by ${ }^{1} \mathrm{H}$ NMR spectroscopy and by GPC (Figures S8 and S9, respectively). We note that the fast response time likely is due to solvent effects. ${ }^{6 b, 20}$ In this case, no induction period is observed, suggesting that the detection event may be faster than the depolymerization reaction. In the absence of added palladium(0) (Figure S10), no depolymerization is detected by ${ }^{1} \mathrm{H}$ NMR over the course of $7 \mathrm{~d}$ at $23{ }^{\circ} \mathrm{C}$. Likewise, control polymer 16 shows no depolymerization when exposed to $\mathrm{Pd}\left(\mathrm{PPh}_{3}\right)_{4}\left({ }^{1} \mathrm{H}\right.$ NMR analysis, Figure S12; GPC analysis, Figure S13).

The polymers are bench stable and only begin to decompose when heated above $150{ }^{\circ} \mathrm{C}$ (Figure S15). All three $n$-butylsubstituted polymers (14-16) display nearly identical thermal gravimetric analysis (TGA) traces between $150{ }^{\circ} \mathrm{C}$ and 180 ${ }^{\circ} \mathrm{C}$, with initial sharp weight losses of $25 \mathrm{wt} \%$ over this $30{ }^{\circ} \mathrm{C}$ temperature range. The $25 \%$ weight loss is consistent with the weight fraction of carbon dioxide that would be released from the polymers upon complete depolymerization. Heating the samples above $180{ }^{\circ} \mathrm{C}$ ultimately results in complete mass loss of the material.

Polymer 15 provided the opportunity to probe the mechanism of depolymerization by looking for spectroscopic indicators of azafulvene $\mathbf{1 8}$ (Figure 3a). Triggered depolymerization of $\mathbf{1 5}$ is achieved in dry dichloromethane (DCM) by addition of dry $\mathrm{Pd}\left(\mathrm{PPh}_{3}\right)_{4}$; no nucleophiles are present to react with the presumed azafulvene depolymerization product. Under these conditions, low-resolution mass data (positive ionization) are consistent with azafulvene 18 (Figure 3b). Exposure of the depolymerization solution to an excess $(5 \mathrm{~mL})$ of dry methanol for $1 \mathrm{~h}$ at room temperature, however, yielded a mass consistent with the methanol adduct of azafulvene (19) as well as unreacted azafulvene (Figure 3c). These results suggest that the depolymerization mechanism proceeds through the release of azafulvene, which then traps nucleophiles in the reaction mixture, much like azaquinone methide reacts with water and other nucleophiles when poly(phenyl carbamate)s depolymerize.

The ease with which derivatives of poly(carboxypyrrole)s are generated is a useful feature of these polymers, both for 

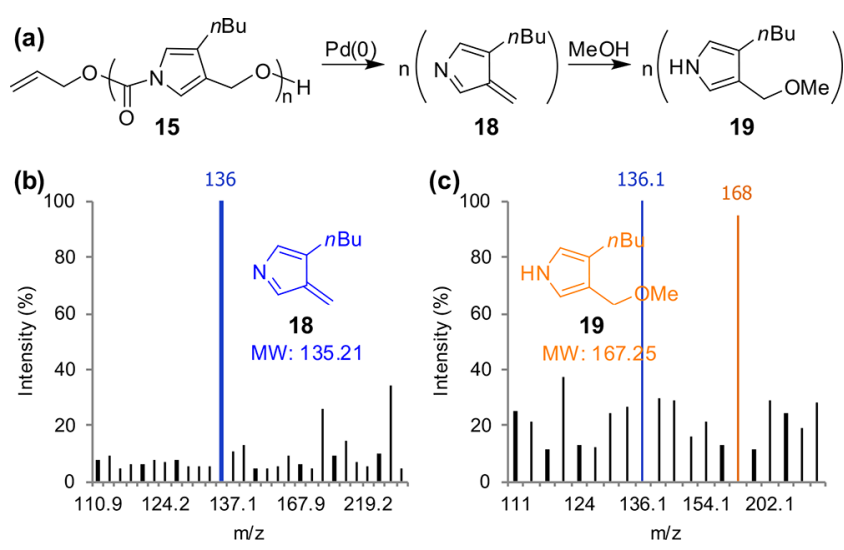

Figure 3. Investigation of the proposed azafulvene depolymerization products. (a) The polymer and reaction used to detect azafulvene. Mass spectra of (b) the depolymerization solution of 15 showing a mass for azafulvene and (c) the same solution after exposure to methanol.

creating materials and for probing the design strategy that we employed when designing this new class of depolymerizable polymers. Given that we originally hypothesized a relationship between rate of depolymerization and the relative aromaticity of the repeating unit (i.e., low aromaticity for fast depolymerization), we prepared an indole variant of the poly(carboxypyrrole)s (Scheme 3 ). Indole is predicted to be

Scheme 3. Synthesis of Poly(carboxyindole)

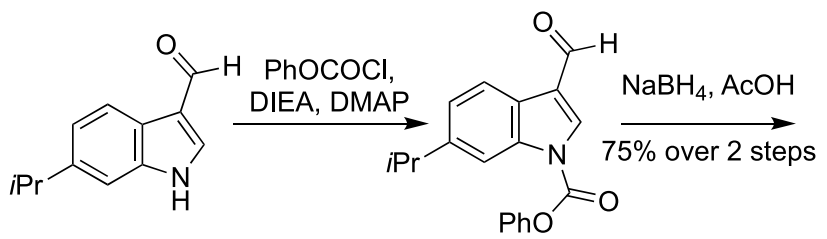

20

21

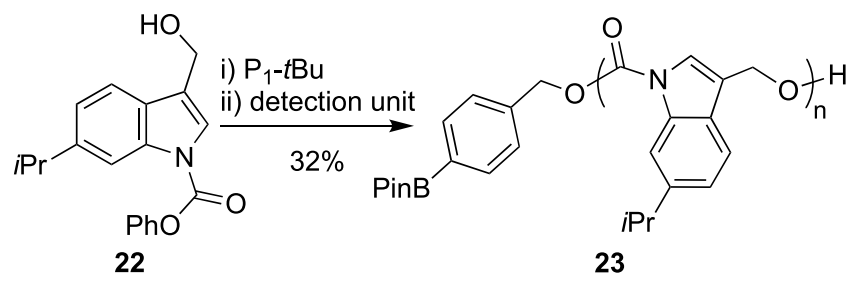

approximately $28 \%$ less aromatic than benzene but $17 \%$ more aromatic than pyrrole (Table S1). On the basis of our aromaticity-depolymerization rate hypothesis, poly(carboxyindole) should depolymerize more slowly than an analogous poly(carboxypyrrole).

We prepared the poly(carboxyindole) in three steps, including attaching the same detection unit present on poly(carboxypyrrole) 14. Exposure of poly(carboxyindole) 23 to hydrogen peroxide under the same conditions as 14 revealed that 23 depolymerizes $12 \times$ slower than 14 , as evidenced by GPC traces (compare Figure 4 with Figures $2 \mathrm{a}$ and S2). This decrease in depolymerization rate for 23 as compared to $\mathbf{1 4}$ is consistent with our proposed relationship between aromaticity of each repeating unit and depolymerization rate, thus suggesting that even faster rates of depolymerization may be possible by further decreasing the

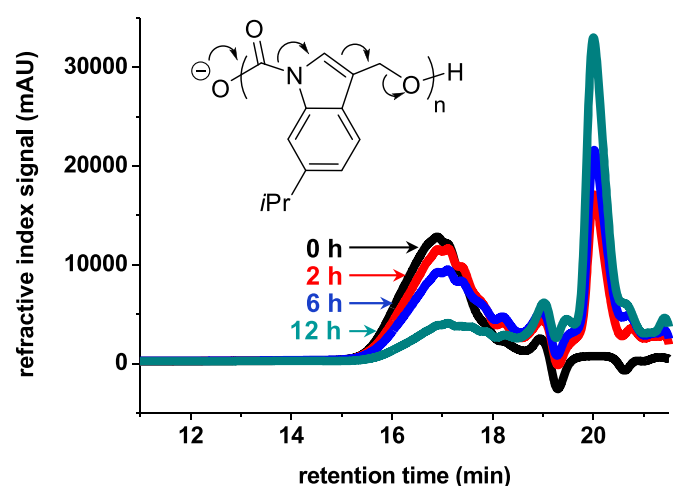

Figure 4. Overlaid GPC chromatograms for poly(carboxyindole) 23 when exposed to 10 equiv of aqueous hydrogen peroxide and triethylamine in THF at $23{ }^{\circ} \mathrm{C}$. The depolymerization reaction was monitored for $12 \mathrm{~h}$.

aromaticity of the repeating units, within the limits of structural stability.

Various types of poly(carboxypyrrole)-based polymeric materials should be readily accessible using the synthetic routes in Scheme 1. Polymer 14, for example, is easily molded into a disc shape using a silicon mold, 40 wt \% poly(ethylene glycol $)\left(M_{\mathrm{n}}=400\right)$ as plasticizer, and DCM as solvent. Evaporation of the solvent yields porous, solid disks (Figure 5).

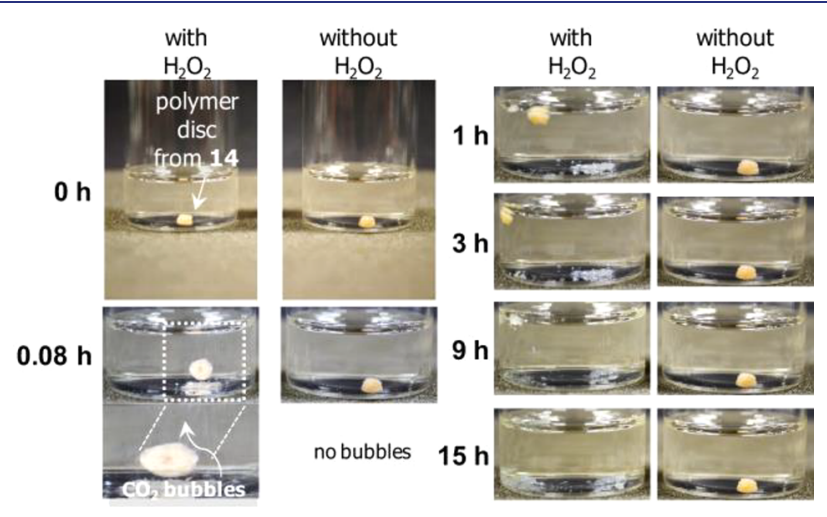

Figure 5. Photographs of solid-state depolymerization of a polymer disc (diameter, $4 \mathrm{~mm}$; thickness, $2 \mathrm{~mm}$ ) from 14 in the presence of triethylamine (10 equiv) and $\mathrm{H}_{2} \mathrm{O}_{2}$ (10 equiv) in $\mathrm{MeCN}(5 \mathrm{~mL})$. The disc was prepared with polymer 14 and PEG plasticizer $\left(M_{n}, 400 ; 40\right.$ wt \%). The control experiment was performed without $\mathrm{H}_{2} \mathrm{O}_{2}$ using the same sample. A compelling video showing a similar response to hydrogen peroxide is available in the Supporting Information.

Exposure of these disks to aqueous hydrogen peroxide (10 $\mathrm{mM})$ and triethylamine $(10 \mathrm{mM})$ in $\mathrm{MeCN}$ results in the rapid formation of bubbles around the disk (Figure 5, inset), which is consistent with depolymerization and generation of carbon dioxide gas. ${ }^{21}$ Within $1 \mathrm{~h}$ of exposure, the disk is noticeably smaller and floats from the bottom of the solution to the airacetonitrile interface (Figure 5). By $9 \mathrm{~h}$, the disk has completely vanished, indicating that triggered depolymerization generated soluble small molecules. A video highlighting this rapid solid-state depolymerization is available in the Supporting Information.

This disappearance of the disk is not caused by dissolution of the polymer in acetonitrile; the polymer is not soluble in this solvent, as evidenced by two control experiments. First, another disk made from $\mathbf{1 4}$ is exposed to acetonitrile 
containing triethylamine but not aqueous hydrogen peroxide (Figure 5). The second control experiment (Figure S14) includes a disk made from polymer 15, which contains a detection unit for palladium $(0)$, immersed in the same hydrogen peroxide solution as in Figure 5. In both controls, no bubbles were evident, and the size of the disks did not change even after $15 \mathrm{~h}$ of exposure to the solutions.

\section{CONCLUSIONS}

In summary, we have designed and synthesized a new class of self-immolative $\mathrm{CD}_{\mathrm{r}}$ polymers. These polymers depolymerize readily in nonpolar solvents and, importantly, depolymerize in the solid state. This last feature is critical for moving forward with attempts to design plastics and other soft materials that can be converted back to small molecules easily and with little input of energy when the plastics are no longer needed. Progress toward this goal is further strengthened by the thermal stability of these poly(carboxypyrrole)s, combined with the ability to customize detection units and applied signals to achieve selective depolymerization. Finally, the synthetic routes to these polymers are straightforward, which should open access to functional derivatives.

\section{ASSOCIATED CONTENT}

\section{(s) Supporting Information}

The Supporting Information is available free of charge at https://pubs.acs.org/doi/10.1021/jacs.0c02774.

Syntheses, characterization data, experimental details, supplementary figures and tables, ${ }^{1} \mathrm{H}$ and ${ }^{13} \mathrm{C}$ NMR spectra, and GPC chromatograms (PDF)

Video S1, highlighting the rapid solid-state depolymerization of a polymer disc in hydrogen peroxide (MP4)

\section{AUTHOR INFORMATION}

\section{Corresponding Authors}

Scott T. Phillips - Micron School of Materials Science and Engineering, Boise State University, Boise, Idaho 83725-2090, United States; 이이이.org/0000-0001-5262-6206; Email: sphillips@boisestate.edu

Hyungwoo Kim - School of Polymer Science and Engineering, Chonnam National University, Buk-gu, Gwangju 61186,

Korea; ○orcid.org/0000-0003-1958-3587; Email: kimhw@ jnu.ac.kr

\section{Authors}

Adam D. Brooks - Micron School of Materials Science and Engineering, Boise State University, Boise, Idaho 83725-2090, United States

Anthony M. DiLauro - Micron School of Materials Science and Engineering, Boise State University, Boise, Idaho 83725-2090, United States

Complete contact information is available at:

https://pubs.acs.org/10.1021/jacs.0c02774

\section{Notes}

The authors declare no competing financial interest.

\section{ACKNOWLEDGMENTS}

This work was supported in part by the U.S. Army Research Office W911NF-14-1-0232 (project contribution: development of polymers that depolymerize in the solid state), W911NF-17-1-0608 (project contribution: development of stimuli-responsive materials using self-immolative polymers), and W911NF-15-1-0351 (project contribution: equipment support).

\section{REFERENCES}

(1) (a) Yardley, R. E.; Kenaree, A. R.; Gillies, E. R. Triggering Depolymerization: Progress and Opportunities for Self-Immolative Polymers. Macromolecules 2019, 52, 6342-6360. (b) Phillips, S. T.; DiLauro, A. M. Continuous Head-to-Tail Depolymerization: An Emerging Concept for Imparting Amplified Responses to StimuliResponsive Materials. ACS Macro Lett. 2014, 3, 298-304. (c) Peterson, G. I.; Larsen, M. B.; Boydston, A. J. Controlled Depolymerization: Stimuli-Responsive Self-Immolative Polymers. Macromolecules 2012, 45, 7317-7328.

(2) (a) Sun, X.; Shabat, D.; Phillips, S. T.; Anslyn, E. V. SelfPropagating Amplification Reactions for Molecular Detection and Signal Amplification: Advantages, Pitfalls, and Challenges. J. Phys. Org. Chem. 2018, 31, e3827. (b) Cho, D.-G.; Sessler, J. L. Modern Reaction-Based Indicator Systems. Chem. Soc. Rev. 2009, 38, 16471662.

(3) Kaitz, J. A.; Lee, O. P.; Moore, J. S. Depolymerizable Polymers: Preparation, Applications, and Future Outlook. MRS Commun. 2015, 5, 191-204.

(4) Roth, M. E.; Green, O.; Gnaim, S.; Shabat, D. Dendritic, Oligomeric, and Polymeric Self-Immolative Molecular Amplification. Chem. Rev. 2016, 116, 1309-1352.

(5) (a) Liu, G.; Zhang, G.; Hu, J.; Wang, X.; Zhu, M.; Liu, S. Hyperbranched Self-Immolative Polymers (hSIPs) for Programmed Payload Delivery and Ultrasensitive Detection. J. Am. Chem. Soc. 2015, 137, 11645-11655. (b) Sagi, A.; Weinstain, R.; Karton, N.; Shabat, D. Self-Immolative Polymers. J. Am. Chem. Soc. 2008, 130, 5434-5435. (c) Lewis, G. G.; Robbins, J. S.; Phillips, S. T. PhaseSwitching Depolymerizable Poly(carbamate) Oligomers for Signal Amplification in Quantitative Time-Based Assays. Macromolecules 2013, 46, 5177-5183. (d) Esser-Kahn, A. P.; Sottos, N. R.; White, S. R.; Moore, J. S. Programmable Microcapsules from Self-Immolative Polymers. J. Am. Chem. Soc. 2010, 132, 10266-10268. (e) Liu, G.; Wang, X.; Hu, J.; Zhang, G.; Liu, S. Self-Immolative Polymersomes for High-Efficiency Triggered Release and Programmed Enzymatic Reactions. J. Am. Chem. Soc. 2014, 136, 7492-7497.

(6) (a) Jung, D.; Lee, K. M.; Tojo, T.; Oh, Y.; Yoon, H.; Kim, H. Dual Cross-Linked Hydrogels that Undergo Structural Transformation via Selective Triggered Depolymerization. Chem. Mater. 2019, 31, 6249-6256. (b) Xiao, Y.; Li, H.; Zhang, B.; Cheng, Z.; Li, Y.; Tan, X.; Zhang, K. Modulating the Depolymerization of SelfImmolative Brush Polymers with Poly(benzyl ether) Backbones. Macromolecules 2018, 51 (8), 2899-2905. (c) Kim, H.; Mohapatra, H.; Phillips, S. T. Rapid, On-Command Debonding of StimuliResponsive Cross-Linked Adhesives by Continuous, Sequential Quinone Methide Elimination Reactions. Angew. Chem., Int. Ed. 2015, 54, 13063-13067. (d) Olah, M. G.; Robbins, J. S.; Baker, M. S.; Phillips, S. T. End-Capped Poly(benzyl ethers): Acid and Base Stable Polymers That Depolymerize Rapidly from Head-to-Tail in Response to Specific Applied Signals. Macromolecules 2013, 46, 5924-5928.

(7) (a) Lutz, J. P.; Davydovich, O.; Hannigan, M. D.; Moore, J. S.; Zimmerman, P. M.; McNeil, A. J. Functionalized and Degradable Polyphthalaldehyde Derivatives. J. Am. Chem. Soc. 2019, 141 (37), 14544-14548. (b) Lee, K. M.; Phillips, O.; Engler, A.; Kohl, P. A.; Rand, B. P. Phototriggered Depolymerization of Flexible Poly(phthalaldehyde) Substrates by Integrated Organic Light-Emitting Diodes. ACS Appl. Mater. Interfaces 2018, 10, 28062-28068. (c) Seo, W.; Phillips, S. T. Patterned Plastics That Change Physical Structure in Response to Applied Chemical Signals. J. Am. Chem. Soc. 2010, 132, 9234-9235. (d) Diesendruck, C. E.; Peterson, G. I.; Kulik, H. J.; Kaitz, J. A.; Mar, B. D.; May, P. A.; White, S. R.; Martinez, T. J.; Boydston, A. J.; Moore, J. S. Mechanically Triggered Heterolytic Unzipping of a Low-Ceiling-Temperature Polymer. Nat. Chem. 2014, 6, 623-628. (e) DiLauro, A. M.; Zhang, H.; Baker, M. S.; Wong, F.; 
Sen, A.; Phillips, S. T. Accessibility of Responsive End-Caps in Films Composed of Stimuli-Responsive, Depolymerizable Poly(phthalaldehydes). Macromolecules 2013, 46, 7257-7265. (f) DiLauro, A.; Robbins, J. S.; Phillips, S. T. Reproducible and Scalable Synthesis of End-Cap-Functionalized Depolymerizable Poly(phthalaldehydes). Macromolecules 2013, 46, 2963-2968. (g) DiLauro, A. M.; Abbaspourrad, A.; Weitz, D. A.; Phillips, S. T. Stimuli-Responsive Core-Shell Microcapsules with Tunable Rates of Release by Using a Depolymerizable Poly(phthalaldehyde) Membrane. Macromolecules 2013, 46, 3309-3313. (h) Hernandez, H. L.; Kang, S.-K.; Lee, O. P.; Hwang, S.-W.; Kaitz, J. A.; Inci, B.; Park, C. W.; Chung, S.; Sottos, N. R.; Moore, J. S.; Rogers, J. A.; White, S. R. Triggered Transience of Metastable Poly(phthalaldehyde) for Transient Electronics. Adv. Mater. 2014, 26, 7637-7642.

(8) (a) Heuchan, S. M.; MacDonald, J. P.; Bauman, L. A.; Fan, B.; Henry, H. A. L.; Gillies, E. R. Photoinduced Degradation of Polymer Films Using Polyglyoxylate-Polyester Blends and Copolymers. ACS Omega 2018, 3, 18603-18612. (b) Fan, B.; Yardley, R. E.; Trant, J. F.; Borecki, A.; Gillies, E. R. Tuning the Hydrophobic Cores of SelfImmolative Polyglyoxylate Assemblies. Polym. Chem. 2018, 9, 26012610. (c) Fan, B.; Salazar, R.; Gillies, E. R. Depolymerization of Trityl End-Capped Poly(Ethyl Glyoxylate): Potential Applications in Smart Packaging. Macromol. Rapid Commun. 2018, 39, 1800173. (d) Fan, B.; Trant, J. F.; Wong, A. D.; Gillies, E. R. Polyglyoxylates: A Versatile Class of Triggerable Self-Immolative Polymers from Readily Accessible Monomers. J. Am. Chem. Soc. 2014, 136, 10116-10123.

(9) (a) Sirianni, Q. E. A.; Rabiee Kenaree, A.; Gillies, E. R. Polyglyoxylamides: Tuning Structure and Properties of SelfImmolative Polymers. Macromolecules 2019, 52, 262-270. (b) Ree, L. H. S.; Sirianni, Q. E. A.; Gillies, E. R.; Kelland, M. A. Systematic Study of Polyglyoxylamides as Powerful, High-Cloud Point Kinetic Hydrate Inhibitors. Energy Fuels 2019, 33, 2067-2075.

(10) (a) DeWit, M. A.; Beaton, A.; Gillies, E. R. A Reduction Sensitive Cascade Biodegradable Linear Polymer. J. Polym. Sci., Part A: Polym. Chem. 2010, 48, 3977-3985. (b) Chen, E. K. Y.; McBride, R. A.; Gillies, E. R. Self-Immolative Polymers Containing Rapidly Cyclizing Spacers: Toward Rapid Depolymerization Rates. Macromolecules 2012, 45, 7364-7374. (c) McBride, R. A.; Gillies, E. R. Kinetics of Self-Immolative Degradation in a Linear Polymeric System: Demonstrating the Effect of Chain Length. Macromolecules 2013, 46, 5157-5166. (d) DeWit, M. A.; Gillies, E. R. A Cascade Biodegradable Polymer Based on Alternating Cyclization and Elimination Reactions. J. Am. Chem. Soc. 2009, 131, 18327-18334.

(11) (a) Joo, W.; Wang, W.; Mesch, R.; Matsuzawa, K.; Liu, D.; Willson, C. G. Synthesis of Unzipping Polyester and a Study of its Photochemistry. J. Am. Chem. Soc. 2019, 141, 14736-14741. (b) Lobez, J. M.; Swager, T. M. Disassembly of Elastomers: Poly(olefin sulfone)-Silicones with Switchable Mechanical Properties. Macromolecules 2010, 43, 10422-10426. (c) Lobez, J. M.; Swager, T. M. Radiation Detection: Resistivity Responses in Functional Poly(Olefin Sulfone)/Carbon Nanotube. Angew. Chem., Int. Ed. 2010, 49, 95-98. (d) Liu, Y.; Jia, Y.; Wu, Q.; Moore, J. S. Architecture-Controlled Ring-Opening Polymerization for Dynamic Covalent Poly(disulfide)s. J. Am. Chem. Soc. 2019, 141, 1707517080. (e) Hernandez, H. L.; Lee, O. P.; Casey, C. M. P.; Kaitz, J. A.; Park, C. W.; Plantz, C. L.; Moore, J. S.; White, S. R. Accelerated Thermal Depolymerization of Cyclic Polyphthalaldehyde with a Polymeric Thermoacid Generator. Macromol. Rapid Commun. 2018, 39, 1800046. (f) Powell, C. R.; Foster, J. C.; Swilley, S. N.; Kaur, K.; Scannelli, S. J.; Troya, D.; Matson, J. B. Self-Amplified Depolymerization of Oligo(thiourethanes) for the Release of COS $/ \mathrm{H}_{2} \mathrm{~S}$. Polym. Chem. 2019, 10, 2991-2995. (g) Watson, C. B.; Tan, D.; Bergbreiter, D. E. Enthalpy-Driven Polyisobutylene Depolymerization. Macromolecules 2019, 52 (8), 3042-3048. (h) Lu, X.-B.; Liu, Y.; Zhou, H. Learning Nature: Recyclable Monomers and Polymer. Chem. - Eur. J. 2018, 24, 11255-11266. (i) Gnaim, S.; Shabat, D. Self-Immolative Chemiluminescence Polymers: Innate Assimilation of Chemiexcitation in a Domino-like Depolymerization. J. Am. Chem. Soc. 2017, 139, 10002-10008. (j) Dahlhauser, S. D.; Escamilla, P. R.; VandeWalle, A.
N.; York, J. T.; Rapagnani, R. M.; Shei, J. S.; Glass, S. A.; Coronado, J. N.; Moor, S. R.; Saunders, D. P.; Anslyn, E. V. Sequencing of Sequence-Defined Oligourethanes via Controlled Self-Immolation. J. Am. Chem. Soc. 2020, 142, 2744-2749. (k) Neary, W. J.; Isais, T. A.; Kennemur, J. G. Depolymerization of Bottlebrush Polypentenamers and their Macromolecular Metamorphosis. J. Am. Chem. Soc. 2019, 141, 14220-14229.

(12) Patrick, J. F.; Robb, M. J.; Sottos, N. R.; Moore, J. S.; White, S. R. Polymers with Autonomous Life-Cycle Control. Nature 2016, 540, 363-370.

(13) (a) DiLauro, A. M.; Lewis, G. G.; Phillips, S. T. Self-Immolative Poly(4,5-dichlorophthalaldehyde) and its Applications in MultiStimuli-Responsive Macroscopic Plastics. Angew. Chem., Int. Ed. 2015, 54, 6200-6205. (b) DiLauro, A. M.; Phillips, S. T. End-Capped Poly(4,5-dichlorophthalaldehyde): A Stable Self-Immolative Poly(aldehyde) for Translating Specific Inputs into Amplified Outputs, Both in Solution and the Solid State. Polym. Chem. 2015, 6, 32523258.

(14) (a) Yeung, K.; Kim, H.; Mohapatra, H.; Phillips, S. T. SurfaceAccessible Detection Units in Self-Immolative Polymers Enable Translation of Selective Molecular Detection Events into Amplified Responses in Macroscopic, Solid-State Plastics. J. Am. Chem. Soc. 2015, 137, 5324-5327. (b) Baker, M.; Kim, H.; Olah, M.; Lewis, G.; Phillips, S. T. Depolymerizable Poly(benzyl ether)-Based Materials for Selective Room Temperature Recycling. Green Chem. 2015, 17, 4541-4545.

(15) Wang, H.-C.; Zhang, Y.; Possanza, C. M.; Zimmerman, S. C.; Cheng, J.; Moore, J. S.; Harris, K.; Katz, J. S. Trigger Chemistries for Better Industrial Formulations. ACS Appl. Mater. Interfaces 2015, 7, 6369-6382.

(16) (a) Robbins, J. S.; Schmid, K. M.; Phillips, S. T. Effects of Electronics, Aromaticity, and Solvent Polarity on the Rate of Azaquinone-Methide-Mediated Depolymerization of Aromatic Carbamate Oligomers. J. Org. Chem. 2013, 78, 3159-3169. (b) Schmid, K. M.; Phillips, S. T. Effect of Aromaticity on the Rate of Azaquinone Methide-Mediated Release of Benzylic Phenols. J. Phys. Org. Chem. 2013, 26, 608-610.

(17) (a) Zhou, Z.; Parr, R. G. New Measures of Aromaticity: Absolute Hardness and Relative Hardness. J. Am. Chem. Soc. 1989, 111, 7371-7379. (b) Hess, B. A., Jr.; Schaad, L. J.; Holyoke, C. W., Jr. On the Aromaticity of Heterocycles Containing the Amine Nitrogen or the Ether Oxygen. Tetrahedron 1972, 28, 3657-3667.

(18) Bossion, A.; Heifferon, K. V.; Meabe, L.; Zivic, N.; Taton, D.; Hedrick, J. L.; Long, T. E.; Sardon, H. Opportunities for Organocatalysis in Polymer Synthesis via Step-Growth Methods. Prog. Polym. Sci. 2019, 90, 164-210.

(19) Cox, P. A.; Reid, M.; Leach, A. G.; Campbell, A. D.; King, E. J.; Lloyd-Jones, G. C. Based-Catalyzed Aryl-B $(\mathrm{OH})_{2}$ Protodeboronation Revisted: From Concerted Proton Transfer to Liberation of a Transient Aryl Anion. J. Am. Chem. Soc. 2017, 139, 13156-13165.

(20) Schmid, K. M.; Jensen, L.; Phillips, S. T. A Self-Immolative Spacer That Enables Tunable Controlled Release of Phenols Under Neutral Conditions. J. Org. Chem. 2012, 77, 4363-4374.

(21) The magnified image is shown in Figure S4 for clarification. 